\title{
Different Effects on Fluid Distribution between Tolvaptan and Furosemide in a Liver Cirrhosis Patient with Chronic Kidney Disease
}

\author{
Izumi Nagayama ${ }^{1}$, Takahiro Masuda ${ }^{1}$, Saki Nakagawa ${ }^{1}$, Takuya Murakami ${ }^{1}$, Ken Ohara $^{1}$, \\ Ryo Matsuoka ${ }^{2}$, Takahisa Kobayashi ${ }^{1}$, Akito Maeshima ${ }^{1}$, Tetsu Akimoto ${ }^{1}$, Osamu Saito ${ }^{1}$, \\ Shigeaki Muto ${ }^{1}$ and Daisuke Nagata ${ }^{1}$
}

\begin{abstract}
:
A 73-year-old man with liver cirrhosis and advanced chronic kidney disease was admitted to our hospital due to bilateral lower leg edema and appetite loss. Furosemide to treat fluid retention markedly decreased extracellular water compared with intracellular water, but the addition of tolvaptan equally decreased both with a greater diuretic response than furosemide alone. Furthermore, tolvaptan administration increased the plasma colloid osmotic pressure, which might facilitate the shift of fluid from the extravascular space to the intravascular space. This is the first case showing different effects on the fluid distribution between furosemide and additional tolvaptan in the same patient.
\end{abstract}

Key words: tolvaptan, furosemide, liver cirrhosis, bioimpedance analysis, extracellular water, intracellular water

(Intern Med 58: 1587-1591, 2019)

(DOI: 10.2169/internalmedicine.2174-18)

\section{Introduction}

Loop diuretics like furosemide have been widely used for the treatment of fluid retention and edema associated with heart failure, renal dysfunction and liver cirrhosis $(1,2)$. However, loop diuretics increase the risk of a worsening renal function and hyponatremia, which are poor prognostic factors (1, 3-5). Tolvaptan, an oral arginine vasopressin V2 receptor antagonist that inhibits water reabsorption in the renal collecting duct, is also used against excess fluid due to congestive heart failure, chronic kidney disease (CKD) and liver cirrhosis (2). This agent can increase the urine volume without decreasing the renal function and activating the sympathetic nervous system and renin-angiotensinaldosterone system (2).

Several studies using bioelectric impedance analyses (BIA), a non-invasive method for assessing the body compo- sition, have shown that the loop diuretic furosemide predominantly lowers the extracellular water (ECW), including the intravascular volume, instead of the intracellular water (ICW) $(6,7)$. However, we recently reported that tolvaptan decreases the ECW and ICW equally without exacerbating the kidney function (8).

No report has yet compared the effects of these two agents on the fluid distribution (ECW and ICW) in the same patient. We therefore evaluated the effects of these two diuretics in a liver cirrhosis patient with advanced CKD.

\section{Case Report}

A 73-year-old man with bilateral lower leg edema and appetite loss was referred and admitted to our hospital. He had been diagnosed with hepatitis $\mathrm{C}$ infection at 38 years of age and liver cirrhosis at 53 years of age. He had received peginterferon alfa 2 a from 63 years of age. The two laparo-

${ }^{1}$ Division of Nephrology, Department of Internal Medicine, Jichi Medical University, Japan, and ${ }^{2}$ Department of Clinical Engineering, Jichi Medical University, Japan

Received: September 22, 2018; Accepted: November 27, 2018; Advance Publication by J-STAGE: February 1, 2019

Correspondence to Dr. Takahiro Masuda, takam@jichi.ac.jp 


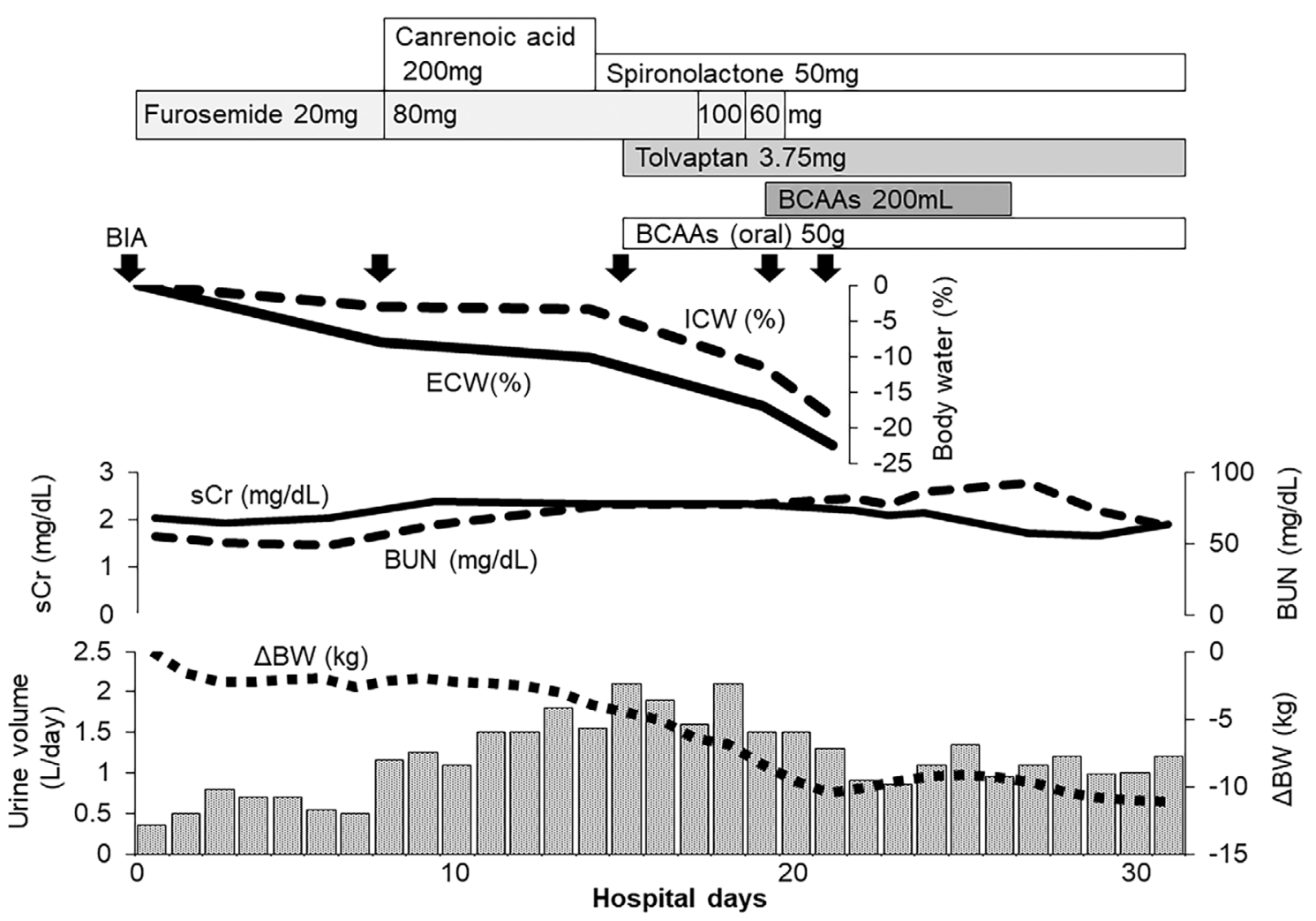

Figure 1. The clinical course and serial changes in the urine volume, blood urea nitrogen (BUN), serum creatinine $(\mathrm{sCr})$, body weight $(\mathrm{BW})$ and intracellular water $(\mathrm{ICW})$ and extracellular water (ECW). BIA: bioelectric impedance analyses, BCAAs: branched-chain amino acids

scopic radiofrequency ablation procedures for hepatocellular carcinoma were performed at 68 and 72 years of age. He had been diagnosed with hypertension at 40 years of age and with CKD [estimated glomerular filtration rate (eGFR) $\left.50-55 \mathrm{~mL} / \mathrm{min} / 1.73 \mathrm{~m}^{2}\right] 3$ years before admission. He developed bilateral lower leg pitting edema and abdominal fullness 3 months before admission, and his primary physician started furosemide (10 mg/day) 1 month later. However, his body weight increased by about $10 \mathrm{~kg}$, so he was referred and admitted to our department.

On admission, he was taking furosemide (10 mg/day), irbesartan (100 mg/day), allopurinol (100 mg/day), amlodipine (5 mg/day) and hydroxyzine (40 mg/day), and peginterferon alfa $2 \mathrm{a}$ was given at $90 \mu \mathrm{g}$ per 3 weeks subcutaneously. He was alert and oriented at the time of admission. An examination showed a body height of $168 \mathrm{~cm}$, weight of $65.7 \mathrm{~kg}$, blood pressure of $120 / 60 \mathrm{mmHg}$ and heart rate of 61 beats/min. Spider angioma was seen on the chest, and the bilateral lower legs were edematous. A laboratory examination revealed the following results: hemoglobin, $11.3 \mathrm{~g} / \mathrm{dL}$; platelet count, $13.2 \times 10^{4} / \mu \mathrm{L}$; prothrombin time-international normalized ratio 1.30 , total bilirubin; 0.86 $\mathrm{mg} / \mathrm{dL}$; aspartate aminotransferase (AST) $28 \mathrm{IU} / \mathrm{L}$; alanine aminotransferase (ALT) 14 IU/L; alkaline phosphatase (ALP) 267 IU/L; $\gamma$-glutamyl transpeptidase ( $\gamma \mathrm{GTP}) 13 \mathrm{U} / \mathrm{L}$; blood urea nitrogen $(\mathrm{BUN}), 55 \mathrm{mg} / \mathrm{dL}$; serum creatinine (sCr), $2.03 \mathrm{mg} / \mathrm{dL}$; eGFR, $26.1 \mathrm{~mL} / \mathrm{min} / 1.73 \mathrm{~m}^{2}$ (CKD stage 4); uric acid $6.9 \mathrm{mg} / \mathrm{dL}$; total protein, $5.6 \mathrm{~g} / \mathrm{dL}$; serum albu- min, $2.2 \mathrm{~g} / \mathrm{dL}$; serum sodium, $142 \mathrm{mEq} / \mathrm{L}$; serum potassium, $5.3 \mathrm{mEq} / \mathrm{L}$; serum chloride, $113 \mathrm{mEq} / \mathrm{L}$; brain natriuretic peptide $99.7 \mathrm{pg} / \mathrm{mL}$; C3 $64 \mathrm{mg} / \mathrm{dL}$ (normal range 86-160 $\mathrm{mg} / \mathrm{dL}$ ); C4 $19 \mathrm{mg} / \mathrm{dL}$ (normal range 17-45 mg/dL); serum IgA $730 \mathrm{mg} / \mathrm{dL}$ (normal range 110-410 $\mathrm{mg} / \mathrm{dL}$ ); urine protein-to-creatinine ratio $1.94 \mathrm{~g} / \mathrm{gCr}$; urine red blood cells 10-19/HPF; urine N-acetyl- $\beta$-D-glucosaminidase (NAG) 37 $\mathrm{U} / \mathrm{gCr}$ (normal range 1.0-6.2 $\mathrm{U} / \mathrm{gCr}$ ) and urine $\beta 2$ microglobulin $<50 \mu \mathrm{g} / \mathrm{L}$. Membranoproliferative glomerulonephritis or IgA nephropathy was assumed as his primary renal disease because of the existence of hepatitis $\mathrm{C}$ infection, moderate proteinuria and hematuria, a mild decrease in the serum complement and an increase in the serum $\operatorname{IgA}$ level. Chest X-ray showed a dull costophrenic angle on the right side without pulmonary congestion, and the cardiothoracic ratio was $47 \%$. Abdominal computed tomography revealed advanced atrophy of the liver with an irregular surface and ascites around the liver. Bilateral renal atrophy was observed (kidney size: right $8.6 \times 4.1 \mathrm{~cm}$, left $8.7 \times 4.2 \mathrm{~cm}$ ). Ultrasound cardiography demonstrated a normal cardiac function (left ventricular ejection fraction: 85\%). His ChildPugh score was 9 points (grade B).

The hospital course is shown in Fig. 1. The daily dose of furosemide $20 \mathrm{mg}$ did not sufficiently increase the urine volume (500-800 mL/day) after 7 days and worsened the renal function (sCr: 2.03 vs. $2.39 \mathrm{mg} / \mathrm{dL}$, BUN: 55 vs. $63 \mathrm{mg} / \mathrm{dL}$, serum uric acid: 6.9 vs. $7.5 \mathrm{mg} / \mathrm{dL}$ ) without increasing the levels of tubular damage marker [urine NAG: 37 vs. $16.8 \mathrm{U} /$ 


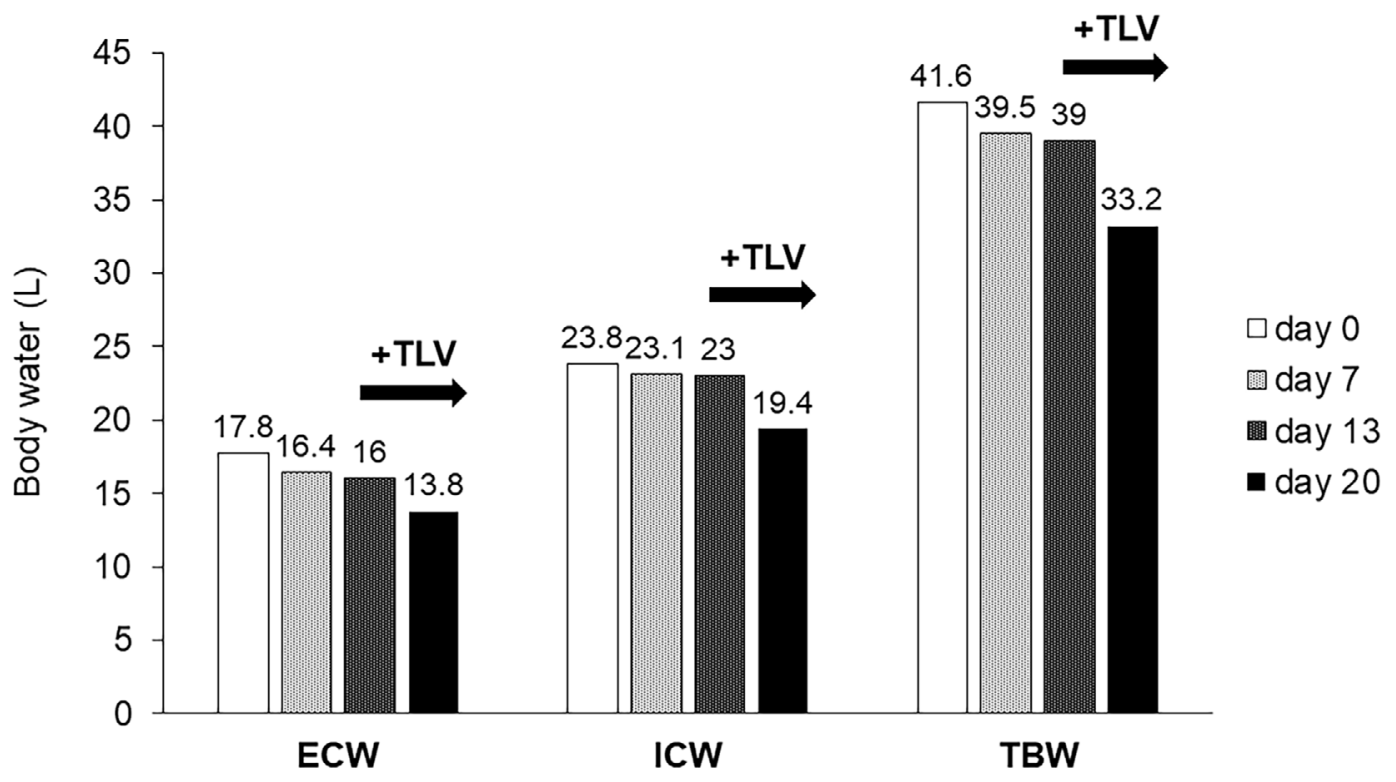

Figure 2. Changes in the extracellular water (ECW), intracellular water (ICW) and total body water (TBW) after admission. The ECW, ICW and TBW were clearly decreased after the initiation of tolvaptan (TLV). Day 0: the day of admission

gCr (Day 4)] (9). The mineralocorticoid receptor (MR) antagonist canrenoic acid was then added (100 mg/day), and furosemide was increased to $80 \mathrm{mg} /$ day on Day 7 . However, the body water as measured by a BIA device (InBody S10; InBody Japan, Tokyo, Japan) $(8,10)$ did not markedly change for a further 6 days (Fig. 1,2) while the BUN and $\mathrm{BUN} / \mathrm{sCr}$ ratio did increase (BUN: 63 vs. $76 \mathrm{mg} / \mathrm{dL}$, BUN/ sCr ratio: 26.4 vs. 32.6), suggesting the existence of mild intravascular dehydration. Canrenoic acid was replaced with another MR antagonist spironolactone (50 mg/day) on Day 12, and tolvaptan (3.75 mg/day) was added on Day 13 . Thereafter, the urine volume increased to around $2,000 \mathrm{~mL} /$ day, and the body weight decreased by about $5 \mathrm{~kg}$ for 7 days without the apparent exacerbation of the renal function (sCr: 2.33 vs. $2.19 \mathrm{mg} / \mathrm{dL}, \mathrm{BUN}$ : 76 vs. $82 \mathrm{mg} / \mathrm{dL}$ ) (Fig. 1). Furthermore, the body water [ECW, ICW and total body water (TBW)] clearly decreased after the initiation of tolvaptan (Fig. 1, 2). Furosemide was discontinued after 5 days of tolvaptan administration (Fig. 1), because the body weight had decreased sufficiently (approximately $5 \mathrm{~kg} / 5$ days) and increased $\mathrm{NH}_{3}(353 \mathrm{ug} / \mathrm{dL})$ with consciousness disturbance and constipation (2 days) were noted. The intravenous supplementation of branched-chain amino acids (BCAAs) $(200 \mathrm{~mL} /$ day) as a treatment for hepatic encephalopathy quickly reduced the $\mathrm{NH}_{3}$ level the next day $(136 \mu \mathrm{g} /$ $\mathrm{dL}$ ), and the $\mathrm{NH}_{3}$ level remained below $200 \mu \mathrm{g} / \mathrm{dL}$ under treatment with tolvaptan and spironolactone. During the 8day intravenous supplementation of BCAAs, the BUN increased from 78 to $93 \mathrm{mg} / \mathrm{dL}$, while the sCr decreased from 2.35 to $1.71 \mathrm{mg} / \mathrm{dL}$ (Fig. 1). After switching to only oral BCAA supplementation ( $50 \mathrm{~g} /$ day), the BUN returned to 62 mg/dL (Day 30) (Fig. 1).

The bioelectrical impedance analysis (BIA) showed that furosemide predominantly decreased the ECW over the ICW
$(-7.9 \%$ vs. $-2.9 \%)$ for the first 7 days (Fig. 3A), while the administration of tolvaptan decreased the ECW and ICW similarly and to a greater degree than furosemide alone ($13.8 \%$ vs. $-15.7 \%$ ) (Fig. 3A). Consequently, the ratio of ECW to TBW (ECW/TBW) was decreased by furosemide treatment $(0.429$ vs. 0.416$)$ but slightly increased by adding tolvaptan (0.410 vs. 0.416) for 7 days (Fig. 3B). Furthermore, the administration of tolvaptan increased the plasma osmolality (301 vs. $318 \mathrm{mOsm} / \mathrm{kg}$ ) and plasma colloid osmotic pressure (14.8 vs. $21.8 \mathrm{mmHg}$ ) (11) accompanied by an increase in the serum albumin (2.1 vs. $2.6 \mathrm{~g} / \mathrm{dL})$ for 6 days (Fig. 3C). The serum sodium was increased by adding tolvaptan (137 vs. $141 \mathrm{mEq} / \mathrm{L})$ but was decreased by furosemide alone (142 vs. $136 \mathrm{mEq} / \mathrm{L}$ ) for 7 days.

\section{Discussion}

The novel finding of this case is the different effects on the fluid distribution between furosemide and tolvaptan in the same patient, where furosemide predominantly decreases the ECW compared with the ICW while tolvaptan administered together with other diuretics equally decreases the ICW and ECW with a greater diuretic response than furosemide alone. Furthermore, the administration of tolvaptan increased the plasma colloid osmotic pressure, which might facilitate the shifting of fluid from the extravascular space to the intravascular space, while furosemide alone decreased the plasma colloid osmotic pressure.

Several studies have shown that loop diuretics predominantly decrease the ECW compared with the $\operatorname{ICW}(6,7,12)$. However, we recently reported that tolvap$\tan$ induces an equivalent reduction rate of ECW and ICW (8). Thus far, no report has evaluated the differences between furosemide and tolvaptan on fluid distribution in 
A

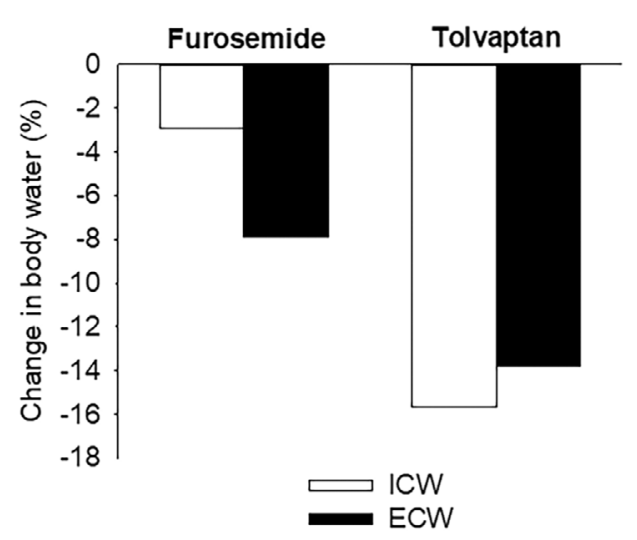

B

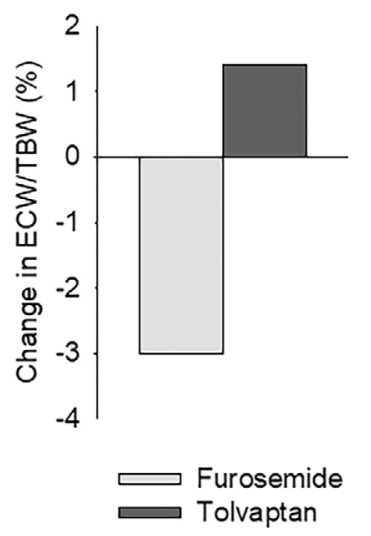

C

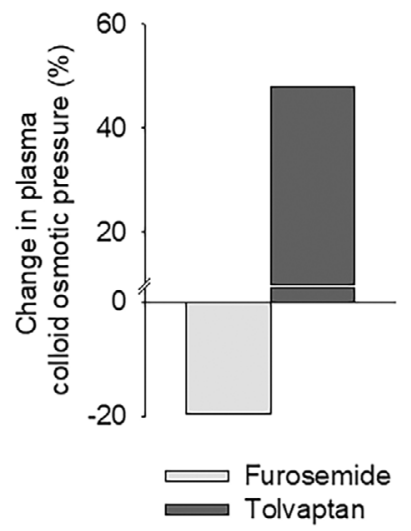

Figure 3. Furosemide predominantly decreased the extracellular water (ECW), but tolvaptan decreased the intracellular water (ICW) and ECW similarly for 7 days from the baseline of each treatment (A). Furosemide decreased the ratio of the ECW to the total body water (TBW) (ECW/TBW) and colloid osmotic pressure, whereas tolvaptan increased both parameters (B and $\mathrm{C}$ ).

the same patient. Generally, a major driving force of fluid movement from the extravascular to the intravascular compartment is the oncotic pressure gradient (13). A recent review proposed that loop diuretics induce a rapid reduction in the intravascular volume and pressure diminishes the intracapillary hydrostatic pressure (13). This may be either a transient or an ongoing imbalance in fluids residing in the intravascular and extravascular compartments, which results in the plasma refill rate being exceeded (13). In contrast, the review proposed that a vasopressin V2 antagonist would increase the oncotic pressure in response to free-water diuresis, thereby leading to the movement of fluid from the interstitial to the intravascular compartment (13). In the present case, an increase in the plasma colloid osmotic pressure $(+47.9 \%)$ was seen 6 days after tolvaptan administration, which might have been the driving force of the fluid shifting from the interstitial to the intravascular compartment.

In addition, the administration of tolvaptan increased the serum sodium concentration, a major determinant of tonicity (effective osmolality) (14), which might have induced the shifting of fluid from the intracellular to the interstitial space, although furosemide alone decreased the serum sodium concentration for the first week. Consequently, the increases in the plasma colloid osmotic pressure and serum sodium concentration after tolvaptan administration might have contributed to the equivalent reduction in the ECW and ICW in this case. It was recently reported that the sugarprotein glycocalyx, which covers the endothelium, plays an important role in the maintenance of an oncotic gradient across the endothelial barrier (15). Thus, a further examination may be necessary to determine whether or not glycocalyx contributes to the differences in the effects on fluid distribution between furosemide and tolvaptan.

The present patient was a responder to tolvaptan, showing a reduction in body weight of approximately $5 \mathrm{~kg} / \mathrm{week}$, which more than matches the "responder criteria" (cut-off value for body weight loss of $1.5 \mathrm{~kg} /$ week) (16). Tolvaptan is reportedly effective for ameliorating fluid retention in patients with liver cirrhosis with ascites $(17,18)$ and advanced CKD $(8,19,20)$, but a low serum sodium level $(<130 \mathrm{mEq} /$ L) is associated with non-responsiveness to tolvaptan (18). In addition, recent studies have shown that tolvaptan exerts a diuretic action even in patients with low serum albumin levels $(18,21,22)$. Because tolvaptan blocks the vasopressin V2 receptor from the basolateral side of the tubules, it is not dependent on the GFR provided the renal blood flow (RBF) is maintained (2). Furthermore, unlike furosemide, tolvaptan exerts diuretic action without binding to albumin, so hypoalbuminemia does not cause diuretic resistance when tolvaptan is given (2). The present patient had liver cirrhosis and advanced CKD (stage 4) with normal serum sodium and low serum albumin levels, so he was expected to be a good responder to tolvaptan. Indeed, the clear diuretic response for tolvaptan might have contributed to the increase in the serum albumin level and plasma colloid osmotic pressure, acting as a driving force for the fluid shift from the interstitial to the intravascular compartment. However, as shown in this case, the careful monitoring and management of excessive diuresis and resultant hepatic encephalopathy is necessary, especially in patients who show a good response to tolvap$\tan$.

A potential limitation in the present case is that the effect of tolvaptan alone on the fluid distribution remains unclear, as tolvaptan was added after treatment with the diuretics furosemide and spironolactone. Similar to the present case, we previously experienced 2 cases in which tolvaptan was administered in addition to furosemide and spironolactone; there as well, tolvaptan equally decreased the ECW and ICW (-5.9\% vs. $-5.3 \%)$ (8). Furthermore, tolvaptan added to a loop diuretic without spironolactone also exerted roughly the same reductive effect on the ECW and ICW in our previous cases $(-7.6 \%$ vs. $-7.0 \%, p=0.425)(8)$. As drug use 
regulations in Japan do not permit monotherapy with tolvap$\tan$ for the treatment of fluid retention, the effects of tolvaptan monotherapy, such as in autosomal dominant polycystic kidney disease patients and/or a head-to-head animal study of furosemide and tolvaptan using a BIA device (22), must be clarified. In addition, the single effect of spironolactone on fluid distribution must also be evaluated, as some interaction between spironolactone and other diuretics may exist.

In conclusion, this case highlights the different effects on the fluid distribution between furosemide and additional tolvaptan in the same patient, where furosemide predominantly decreases the ECW compared with the ICW while tolvaptan administration equally decreases the ICW and ECW with a greater diuretic response than furosemide alone. Furthermore, the administration of tolvaptan increased the plasma colloid osmotic pressure, which might facilitate shifting fluid from the extravascular space to the intravascular space.

This case was presented at the $47^{\text {th }}$ Eastern Regional Meeting of the Japanese Society of Nephrology.

The authors state that they have no Conflict of Interest (COI).

\section{References}

1. Cleland JG, Coletta A, Witte K. Practical applications of intravenous diuretic therapy in decompensated heart failure. Am J Med 119: S26-S36, 2006.

2. Mori T, Ohsaki Y, Oba-Yabana I, Ito S. Diuretic usage for protection against end-organ damage in liver cirrhosis and heart failure. Hepatol Res 47: 11-22, 2017.

3. Damman K, Navis G, Voors AA, et al. Worsening renal function and prognosis in heart failure: systematic review and metaanalysis. J Card Fail 13: 599-608, 2007.

4. Hirano T, Yamamura Y, Nakamura S, Onogawa T, Mori T. Effects of the V(2)-receptor antagonist OPC-41061 and the loop diuretic furosemide alone and in combination in rats. J Pharmacol Exp Ther 292: 288-294, 2000.

5. Matsue Y, Suzuki M, Seya M, et al. Tolvaptan reduces the risk of worsening renal function in patients with acute decompensated heart failure in high-risk population. J Cardiol 61: 169-174, 2013.

6. Vasavada N, Agarwal R. Role of excess volume in the pathophysiology of hypertension in chronic kidney disease. Kidney Int 64 : 1772-1779, 2003.

7. Soderberg M, Hahn RG, Cederholm T. Bioelectric impedance analysis of acute body water changes in congestive heart failure. Scand J Clin Lab Invest 61: 89-94, 2001.

8. Masuda T, Murakami T, Igarashi Y, et al. Dual impact of tolvaptan on intracellular and extracellular water in chronic kidney disease patients with fluid retention. Intern Med 55: 2759-2764, 2016.
9. Masuda T, Akimoto T, Ando Y, et al. Changes in the urinary excretion of beta2-microglobulin (beta 2MG) and N-acetyl-beta-Dglucosaminidase (NAG) during treatment for lupus nephritis. Intern Med 47: 287-290, 2008.

10. Masuda T, Ohara K, Murakami T, et al. Sodium-glucose cotransporter 2 inhibition with dapagliflozin ameliorates extracellular volume expansion in diabetic kidney disease patients. POJ Diabetes Obes 1: 1-8, 2017.

11. Nitta S, Ohnuki T, Ohkuda K, Nakada T, Staub NC. The corrected protein equation to estimate plasma colloid osmotic pressure and its development on a nomogram. Tohoku J Exp Med 135: 43-49, 1981.

12. Ng Kam Chuen MJ, Lip GY, Macfadyen RJ. Performing repeated noninvasive bedside measures of volume response to intravenous furosemide in acute pulmonary edema: a feasibility assessment. Cardiovasc Ther 27: 89-95, 2009.

13. Goldsmith SR, Bart BA, Burnett J. Decongestive therapy and renal function in acute heart failure: time for a new approach? Circ Heart Fail 7: 531-535, 2014.

14. Stookey JD, Purser JL, Pieper CF, Cohen HJ. Plasma hypertonicity: another marker of frailty? J Am Geriatr Soc 52: 1313-1320, 2004.

15. Okada H, Takemura G, Suzuki K, et al. Three-dimensional ultrastructure of capillary endothelial glycocalyx under normal and experimental endotoxemic conditions. Crit Care 21: 261, 2017.

16. Hiramine $Y$, Uojima $H$, Nakanishi $H$, et al. Response criteria of tolvaptan for the treatment of hepatic edema. J Gastroenterol 53: 258-268, 2018

17. Kogiso T, Kobayashi M, Yamamoto K, et al. The outcome of cirrhotic patients with ascites is improved by the normalization of the serum sodium level by tolvaptan. Intern Med 56: 2993-3001, 2017.

18. Hayashi M, Abe K, Fujita M, Okai K, Takahashi A, Ohira H. Association between the serum sodium levels and the response to tolvaptan in liver cirrhosis patients with ascites and hyponatremia. Intern Med 57: 2451-2458, 2018.

19. Katsumata M, Hirawa N, Sumida K, et al. Effects of tolvaptan in patients with chronic kidney disease and chronic heart failure. Clin Exp Nephrol 21: 858-865, 2017.

20. Tominaga $N$, Kida $K$, Inomata $T$, et al. Comparison of the effects of tolvaptan and furosemide on renal water and sodium excretion in patients with heart failure and advanced chronic kidney disease: a subanalysis of the K-STAR study. Clin Exp Nephrol 2018(Epub ahead of print) .

21. Takada T, Masaki T, Hoshiyama A, Toki T, Kamata Y, Shichiri M. Tolvaptan alleviates excessive fluid retention of nephrotic diabetic renal failure unresponsive to furosemide. Nephrology (Carlton) 23: 883-886, 2018.

22. Takagi K, Sato N, Ishihara S, et al. Effects of tolvaptan on urine output in hospitalized heart failure patients with hypoalbuminemia or proteinuria. Heart Vessels 33: 413-420, 2018.

The Internal Medicine is an Open Access journal distributed under the Creative Commons Attribution-NonCommercial-NoDerivatives 4.0 International License. To view the details of this license, please visit (https://creativecommons.org/licenses/ by-nc-nd/4.0/).

(C) 2019 The Japanese Society of Internal Medicine Intern Med 58: 1587-1591, 2019 